\title{
Classification of Cell Cycle Phases in 3D Confocal Microscopy Using PCNA and Chromocenter Features
}

\author{
Stefan Jaeger, \\ Kannappan Palaniappan \\ Department of Computer Science \\ University of Missouri \\ Columbia, MO 65211, USA \\ \{jaegers, pal\}@missouri.edu
}

\author{
Corella S. Casas-Delucchi, \\ M. Cristina Cardoso \\ Department of Biology \\ Technische Universität Darmstadt \\ 64287 Darmstadt, Germany \\ \{casas, cardoso\}@bio.tu-darmstadt.de
}

\begin{abstract}
Cell cycle progression studies using subcellular markers offer important insight into cellular mechanisms of disease and therapeutic drug development. Due to the large volumes of microscopy data involved in such studies, a manual approach to extracting quantitative information is not only prohibitive but error prone. We present an automatic cell cycle phase identification algorithm applied to 3D spinning disk confocal microscopy imagery of mouse embryonic fibroblast cells. In our training dataset, each 3D image stack depicts a single cell in a manually identified cell phase, and is recorded via two channels showing the fluorescently marked protein PCNA and the chromocenters, respectively. We use a 3D $\mathrm{k}$-means approach to segment each volume and extract a set of shape and curvature features to characterize the subcellular foci patterns associated with cell cycle phases for each channel. Radial features are used to describe the spatial distribution of PCNA over the course of the cell cycle. A support vector machine (SVM) classifier using 234 features was trained and achieved a recognition rate of $83 \%$ for the chromocenter and $86 \%$ for the PCNA channels separately on the testing data. A combined SVM classifier using both channels and 468 features further improved the accuracy to nearly $92 \%$ for five phases (G1, SE, SM, SL, G2) and shows promising scalability.
\end{abstract}

\section{Keywords}

Fluorescence microscopy, confocal microscopy, cell cycle analysis, cell cycle phase classification, blobbiness features, cell segmentation

\section{INTRODUCTION}

Accurate measurement of cell cycle progression is useful for understanding disease processes such as cancer and high-

* Corresponding author

Permission to make digital or hard copies of all or part of this work for personal or classroom use is granted without fee provided that copies are not made or distributed for profit or commercial advantage and that copies bear this notice and the full citation on the first page. To copy otherwise, to republish, to post on servers or to redistribute to lists, requires prior specific permission and/or a fee.

ICVGIP'10, December 12-15, 2010, Chennai, India

Copyright 2010 ACM 978-1-4503-0060-5/10/12 ...\$10.00. throughput anti-cancer drug screening studies $[1,3,9,20$, $25]$. Quantifying changes in the cell cycle phases between untreated and treated cells offer a promising screening approach for discovering active compounds for new effective drug development. Our main motivation and long-term interest lies in the effects of different epigenetic modifications on the replication of specific regions of the genome $[15,24$, $16,9,3,14]$. This was studied by inducing epigenetic modifications using a drug and then observing the effects on replication timing using live cell microscopy, labeling both the regions of interest as well as replication sites. Our immediate objective for these data is twofold: First, we want to learn how the treatment changes the (relative) duration of each cell cycle phase, including the subphases of S-phase (SE/SM/SL) [11, 12]. Second, we want to find out if the treated cells show increased phase dependent colocalization of active chromocenter regions with proliferating cell nuclear antigen (PCNA) protein foci that mark sites of active DNA synthesis during replication $[6,23]$.

The biological details and replication timing are described elsewhere and we focus exclusively on the accurate identification of cell cycle phases using feature extraction and classifier design. An automated approach to this is preferable because live cell microscopy experiments in practice typically produce large amounts of data with thousands of cells that are prohibitive to evaluate manually $[17,8,7]$. We extend our previous approaches to cell cycle phase identification by using 3D stacks (instead of only 2D imagery) and two fluorescence channels (chromocenters and PCNA instead of just PCNA) $[9,3]$.

We structured the paper as follows: Section 2 describes the biological background and the data used in our experiments. Section 3 shows how we segment individual cells and compute features. Finally, Section 4 presents our classification results followed by conclusions.

\section{BIOLOGICAL MOTIVATION}

A spinning disk confocal microscope captures live mouse cells in various cell cycle phases every $30 \mathrm{~min}$ for several hours (typically between 10 and 40 hours). With a spinning disk confocal microscope, which is kind of a multipoint confocal microscope, we can achieve both high resolutions and high-throughput data rates. The stacks have an XY resolution of $100 \mathrm{~nm}$ and a $\mathrm{z}$-step of $500 \mathrm{~nm}$. Each stack contains slices with $1000 \times 1000$ voxels, where the number of slices (z-steps) varies per experiment. 

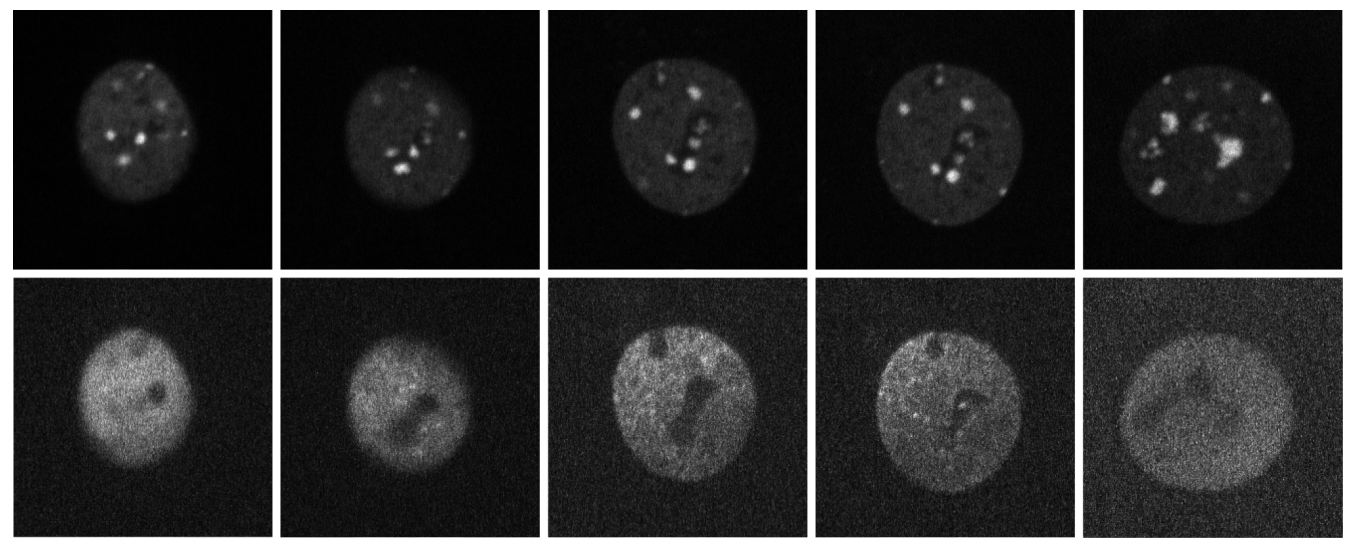

Figure 1: Middle slices of cells in G1, SE, SM, SL, and G2 phases (from left to right). The top row shows the chromocenters and the bottom row the replication structures (PCNA).

A replicating cell typically undergoes the following cell cycle: After the cell divides (mitosis/ M-phase), it enters the G1-phase. The first column of Figure 1 shows an example of a cell in G1 phase. Following the G1-phase, after completing a series of "tasks" such as cell growth, the cell starts preparing for the next division by duplicating its DNA, which marks the beginning of S-phase. This is when we actually start seeing structures or replication foci. Sphase progresses from early S-phase (SE) to mid S-phase (SM) and late S-phase (SL). When the cell has duplicated its entire DNA, it enters G2-phase to begin cytokinesis (see Figure 1).

Fluorescent markers related to chromocenter and replication regions were used in the experiments. The chromocenters are structures built around specific regions of DNA (the major satellite sequences), while the replication structures are foci of fluorescently labeled PCNA/ RFP-PCNA, a protein involved in DNA replication and repair. The visual appearance of the chromocenters in the first channel is relatively stable throughout the different stages of the cell cycle, until the G2-phase. At the end of G2, chromosomes start condensing for mitosis. When this happens (prometaphase), chromosomes can be seen as individual entities. At this point the chromocenters, which are usually clusters of heterochromatin, change their shape and appear as elongated objects (see last column of top row in Figure 1). The distribution of PCNA foci is uniform during G1-phase and G2-phase, much diluted during M-phase, and shows a complex temporally varying distribution of foci patterns during different stages of S-phase [15, 24]. As soon as we start seeing welldefined sub-cellular structures, i.e. small foci distributed throughout the cell, we say early S-phase has started. At this point, the nucleolar and nucleolar rim are rather free of replication regions. Then, as replication structures begin to populate these regions, mid S-phase starts. The beginning of the replication of the chromocenters marks the start of late S-phase, with pronounced foci in the nucleolar rim.

In terms of cell cycle phase discrimination, the subphases of S-phase are the hardest to discriminate due to the difficulty in accurately distinguishing between the PCNA foci distribution patterns, particularly between SE and SM [9]. In addition, these phases can often be confused with a socalled "very late" S-phase, which starts when the chromocenters have almost finished replicating and there are still a few smaller foci distributed in the nucleus. This is when the last unreplicated DNA pieces start replicating before G2, mostly nearby the chromocenters.

Given the subtle temporally and spatially varying structures in the PCNA channel, the classification of cell cycle phases is a challenging image analysis and machine learning problem. To solve the classification problem, we proceed in three steps: First, we segment the individual cell nuclei from each image volume. Then, we compute features for both channels of each segmented nucleus. Finally, we derive histogram features as input to an SVM classifier.

\section{3D SEGMENTATION AND 3D FEATURES}

The image volume is first segmented into cell (foreground) and non-cell (background) regions. Following segmentation a large set of descriptive features are extracted to describe the spatial distribution of brightness foci patterns during different stages of the cell cycle. A machine learning multiclass discrimination algorithm is then applied to the feature set to distinguish between different cell cycle phases.

\subsection{D Segmentation}

Segmentation in live cell imaging has been the subject of intensive research in recent years. Our group and others have extensively investigated the applicability and robustness of using active contour level-set methods for cell segmentation in bioimaging time lapse microscopy applications $[3,4,20,18,22]$. In this work we tested a modified $\mathrm{k}$-means clustering approach with adaptive thresholding to extract individual cells. This approach works fairly well on 3D microscopy imagery with the clusters representing the different intensities of nuclei and background in our case. In particular, we modified the standard k-means method given by the expression

$$
\underset{S}{\operatorname{argmin}} \sum_{i=1}^{k} \sum_{x_{j} \in S_{i}}\left\|x_{j}-\mu_{i}\right\|^{2}
$$

where the $x_{j}$ represent the different voxel intensities and $\mu_{i}$ stands for the mean of cluster $S_{i}$. In our modified k-means method, the clusters of the final cell segmentation are given by

$$
S_{\text {seg }}=\left\{S_{i} \mid \bar{S}_{i} \geq \gamma * \max _{i}\left(\bar{S}_{i}\right)\right\}
$$


with $0 \leq \gamma \leq 1$, where $\bar{S}_{i}$ denotes the average intensity of all pixels belonging to cluster $S_{i}$ and $\max _{i}\left(\bar{S}_{i}\right)$ is the maximum of all these averages. The idea is to make kmeans more robust against noise by using trimmed intensity averages across multiple clusters. In our experiments, we set $\gamma=0.5$. The resulting segmentation can be used to initialize a 3D level set active contour algorithm such as fast graph partitioning active contours $[2,19]$ to further improve the quality of the cell boundary surfaces.

\subsection{D Multichannel Feature Estimation}

We follow a bag-of-features approach and compute a highdimensional set of features for each channel [21]. This includes the following features:

\section{I: Voxel intensity histograms}

GM: 3D gradient magnitude histograms

A1,A3: As 3D shape descriptors, we use the following two shape indices

$$
\begin{gathered}
A 1=\tan ^{-1}\left(\frac{\lambda_{1}}{\lambda_{2}}\right) \\
A 3=\cos ^{-1}\left(\frac{\lambda_{3}}{\sqrt{\lambda_{1}^{2}+\lambda_{2}^{2}+\lambda_{3}^{2}}}\right)
\end{gathered}
$$

where $\lambda_{1}, \lambda_{2}, \lambda_{3}$ are the eigenvalues of the Hessian with $\lambda_{1} \leq \lambda_{2} \leq \lambda_{3}$.

A2: As a $3 \mathrm{D}$ curvature shape descriptor, we use the angle measure

$$
A 2=\tan ^{-1}\left(\frac{\sqrt{\lambda_{1}^{2}+\lambda_{2}^{2}+\lambda_{3}^{2}}}{1+I(x, y, z)}\right)
$$

where $I(x, y, z)$ denotes the voxel intensity at position $(x, y, z)$. This feature is bounded by $0 \leq \gamma \leq \pi / 2$ and is independent of image brightness.

EV: The angle between the $\mathrm{x}$-axis and the eigenvector associated with the largest eigenvalue of the local image volume Hessian.

HOG: In addition to the edge-based magnitude histogram (GM) we also use the 3D edge orientation histogram descriptor - the histogram of oriented gradients (HOG). Histogram of oriented gradients are feature descriptors that have been successfully used in many recent object detection and people detection applications [5, 21]. HOG uses the histogram of gradient orientations weighted by corresponding gradient magnitudes over a patch or small region of an image.

R: 3D radial distribution of brightness foci features that were used to measure the degree of co-localization are beneficial for distinguishing between the sub-phases of S-phase (SE, SM, and SL) [12]. We compute radial features by first detecting sub-cellular blobs or foci in the different channels. For detecting blobs, which represent either chromocenters or PCNA in our application, we follow the approach of Frangi et al. [10, 13]. They use a multi-scale approach that analyzes the eigenvalues of the Hessian matrix.
A typical way to describe the local behavior of an image $I$ is to consider its Taylor expansion in the neighborhood of a point $x_{o}$ (up to the second order):

$$
I\left(x_{o}+\delta_{x_{o}}, s\right) \approx I\left(x_{o}, s\right)+\delta x_{o}^{T} \nabla_{o, s}+\delta x_{o}^{T} \mathcal{H}_{o, s} \delta x_{o}
$$

where $\nabla_{o, s}$ and $\mathcal{H}_{o, s}$ are the gradient vector and Hessian matrix of the image computed in $x_{o}$ at scale $s$. In the framework of Frangi et al., differentiation is defined as a convolution with derivatives of Gaussians [10]:

$$
\frac{\partial}{\partial x} I(x, s)=s^{\gamma} I(x) * \frac{\partial}{\partial x} G(x, s)
$$

where the D-dimensional Gaussian is defined by

$$
G(x, s)=\frac{1}{\sqrt{\left(2 \pi s^{2}\right)}{ }^{D}} e^{-\frac{\|x\|^{2}}{2 s^{2}}}
$$

and the parameter $\gamma$ weights the response of differential operators at multiple scales. When $\gamma$ is set to unity, no scale is preferred.

We use the following measure to detect spherical blobs

$$
B(x, y, z)=\frac{\left|\lambda_{1}(x, y, z)\right|}{\sqrt{\left|\lambda_{2}(x, y, z) \lambda_{3}(x, y, z)\right|}}
$$

where $\lambda_{1}, \lambda_{2}, \lambda_{3}$ are the eigenvalues of the Hessian, with $\left|\lambda_{1}\right| \leq\left|\lambda_{2}\right| \leq\left|\lambda_{3}\right|$. This ratio attains its maximum for a blob-like structure and is zero whenever $\lambda_{1} \approx 0$, or as $\lambda_{2}$ and $\lambda_{3}$ tend to be very large. We analyze this "blobbiness" feature at different scales $s$ (see [26] for more information about spherical tensors). The response will be maximum at a scale that approximately matches the size of the blob. The final measure is then the integrated maximum filter response across different scales.

The chromocenter regions characteristically replicate during the late S-phase, so the degree of colocalization with the replication structures of the PCNA channel in different image regions allows us to check for late Sphase patterns. To quantify the blobbiness in different regions of the image, we compute radial features for the blobbiness measure defined by Eq. 8 for each voxel of the segmented cell. In particular, given the k-means cell segmentation as described above, we compute the i-th radial feature $R_{i}$ for each voxel in the cell volume as follows

$$
R_{i}=\sum_{x} \sum_{y} \sum_{z} d_{i}(x, y, z)
$$

with

$$
d_{i}(x, y, z)= \begin{cases}B(x, y, z) & \text { if distance of }(x, y, z) \text { to } \\ & \text { cell boundary is } i \\ 0 & \text { otherwise. }\end{cases}
$$

where $B(x, y, z)$ is the blobbiness measure for the voxel at position $(x, y, z)$ in the original image $I(x, y, z)$, computed according to Eq. 8, and $d_{i}(x, y, z)$ checks the distance to the cell boundary based on a distance transform computed on the segmented image. If the distance of $(x, y, z)$ to the cell boundary is $i$, then $d_{i}(x, y, z)$ returns $B(x, y, z)$; otherwise, it returns zero. The feature is then normalized so that it becomes independent of brightness. 
Table 1: Data samples for each phase.

\begin{tabular}{|c|c|c|c|c|}
\hline G1 & SE & SM & SL & G2 \\
\hline 82 & 115 & 35 & 54 & 14 \\
\hline
\end{tabular}

We quantize each of the features listed above into 32 histogram bins, with the exception of the radial features, which are binned into 10 histogram cells. We use either the full data range for normalization for the angular features or a stabilized normalization that cuts off outliers at the $5 \%$ and $95 \%$ percentiles before normalization for the intensity features. All features together form an 234-dimensional feature vector. The overall number of features computed for both channels is thus 468 .

\section{EXPERIMENTAL RESULTS}

In the experiments reported here, we use six classes for the k-means segmentation $(\mathrm{k}=6)$ and 10 radii for the radial features. Our database contains 300 image volumes of transfected mouse embryonic fibroblast cells acquired with a PerkinElmer UltraView spinning disc system every 30 minutes for up to 40 hours. Each 3D volume consists of dual fluorescence channels using tagged proteins that label major satellite repetitive DNA sequence structures known as chromocenters (CC) using Ma-SAT GFP and active replication sites using mRFP-PCNA (PCNA). An image stack can consist of up to 50 frames, with the typical size between ten and twenty slices. The size of the slices is either $260 \times 260$ or $300 \times 300$. Table 1 shows the number of samples for each cell phase.

\subsection{Qualitative Results}

Figure 2 and Figure 3 show the histograms for the features described in the previous section, both for chromocenters (Fig. 2) and PCNA (Fig. 3) fluorescence channels. All histograms in both figures are normalized and averaged over the whole data set. Each row contains the histograms for one feature, beginning with the radial features $\mathrm{R}$, and followed by intensity I, gradient magnitude GM, shape and curvature indices A1-A3, the angle of the eigenvector associated with the largest eigenvalue EV, and histogram of gradients HOG. Each column contains the histograms associated with one cell cycle phase, beginning with G1, and followed by SE, SM, SL, and G2. We can see that the differences between histograms for different cell cycle phases can be very subtle. Nevertheless, some features have clearly different histograms between cell cycle phases. For instance, the histograms of the intensity feature for the chromocenter channel (second row in Figure 2) have relatively distinctive distributions for each cell cycle phase. Their general shape and the position of their peaks differ significantly among each cell cycle phase. In addition, the intensity histograms computed for the chromocenters display a distribution that is very different from the distribution in the intensity histograms computed for PCNA.

\subsection{Cell Cycle Phase Recognition Rates}

Table 2 shows the recognition rates for the two-class recognition problems, using a linear-kernel SVM classifier between each pair of classes. All recognition rates in Table 2 are based on 5 -fold cross validation runs. The rates are all rela-
Table 2: Recognition rates for two class problems.

\begin{tabular}{|c|c|c|c|c|}
\hline & G1 & SE & SM & SL \\
\hline G1 & - & - & - & - \\
\hline SE & 97.45 & - & - & - \\
\hline SM & 96.58 & 84.56 & - & - \\
\hline SL & 99.26 & 97.02 & 85.39 & - \\
\hline G2 & 95.83 & 97.66 & 93.88 & 89.71 \\
\hline
\end{tabular}

tively high, except for some difficult cases of SE, SM and SL. This confirms our first visual assessment from above: The discrimination between the subphases of S-phase is indeed harder. For instance, the recognition rates of the SVM classifiers for the phase pairs SM/SE and SL/SM are $84.56 \%$ and $85.39 \%$, respectively, which are the lowest accuracies.

We extended the two-class classification problem to the full five-class problem using a one-vs-all method (see Table 3). We trained a single linear-kernel SVM classifier for each class and assigned unknown patterns to the label of the classifier with the highest confidence value, defined as the class with the largest distance to the separating hyperplane. The overall recognition rate we achieved with this approach on the five-class problem was almost $92 \%$. This rate is based on the entire 468-dimensional feature vector.

We also computed recognition rates for different feature subsets to get a better understanding of the performance of individual features and the contribution of each channel. For example, Table 3 shows the results we achieved on our dataset with leave-one-out evaluation. The columns show the recognition rates for radial features, intensity, gradient magnitude, the indices A1 to A3, the EV feature, and HOG. The first two rows, labeled with $\mathrm{CC}$, show the recognition rates for each individual feature of the first channel and the recognition rate for all features of the first channel together. Analogously, the next two rows show the individual recognition rates for the second PCNA channel, and the recognition rate using all features computed on the second channel. The last row of Table 3 shows the overall recognition rate based on the combined set of features from both fluorescence channels to be about 6 percent or more better than using a single channel.

Table 3 also shows that the combined recognition rate using all of the features is much higher than any of the individual feature recognition rates, showing that the overall classification performance cannot be attributed to a single feature. Similarly, the recognition rates of the individual channels are lower compared to the recognition rate when both channels are used jointly. As expected, the second channel performs better than the first chromocenter channel because the distribution of PCNA assumes different patterns throughout the cell cycle phases. As for the performance of the individual features, we can see that they vary greatly from a low of $30 \%$ (A2, PCNA) to a high of $73 \%$ (EV, PCNA). Some features provide a relatively high performance, such as the intensity and $\mathrm{EV}$ feature for the first channel, and the A1 and the EV feature for the second channel. Other features, however, perform poorly, such as the gradient magnitude and A2 feature for the second channel. It should be noted that the mouse embryonic fibroblast cell datasets used in the current experiments generate foci patterns that are less distinctive and hence harder to classify than the human HeLa 
G1
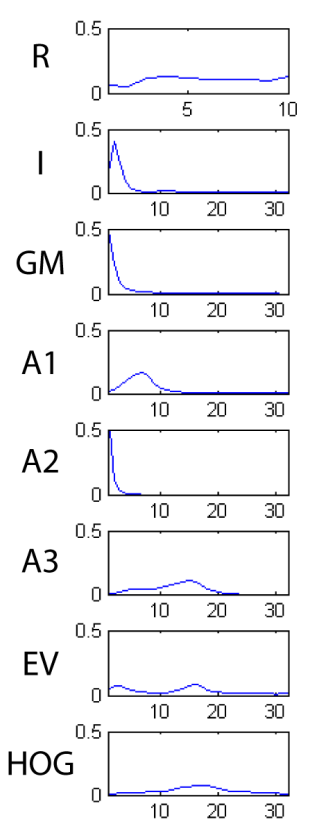

SE
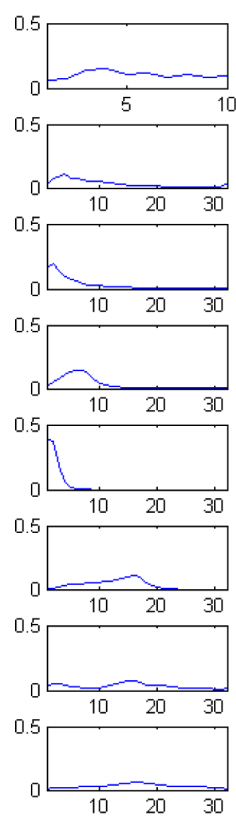

SM
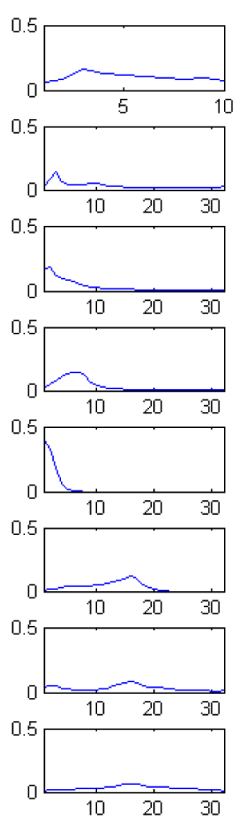

$\mathrm{SL}$
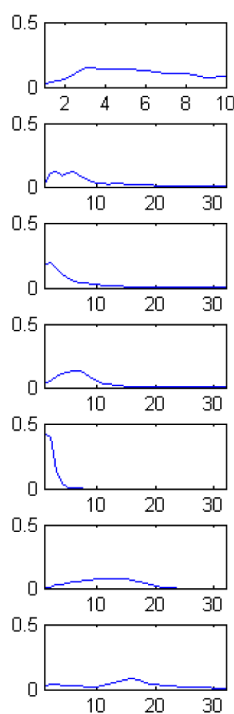

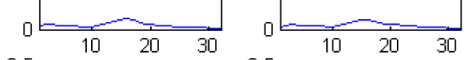

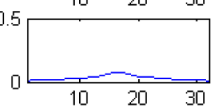

G2
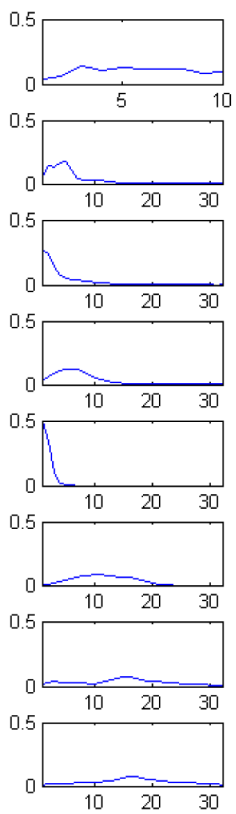

Figure 2: Feature histograms used to construct the 234-dimensional feature vectors are shown for each cell cycle phase for the chromocenter labeled fluorescence channel.

G1
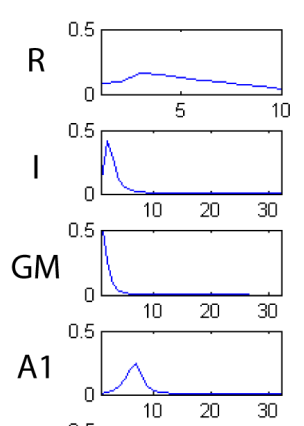

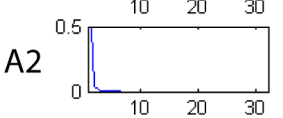
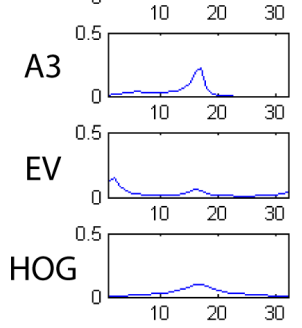

SE
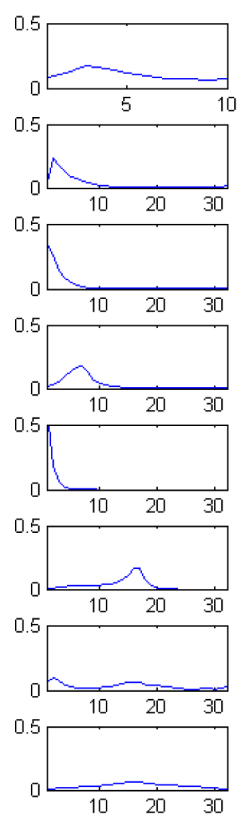

SM
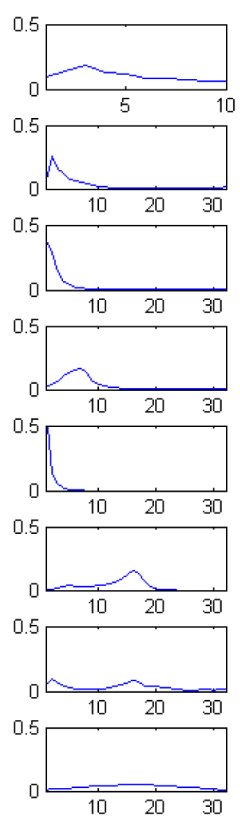

SL
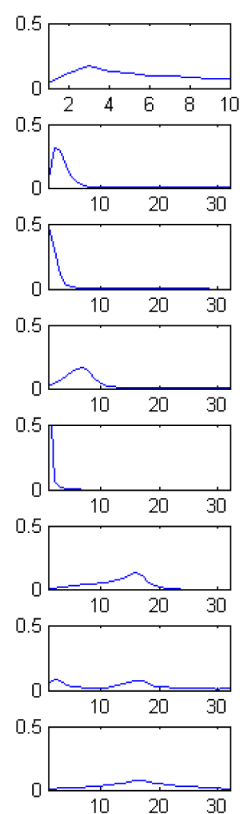

G2
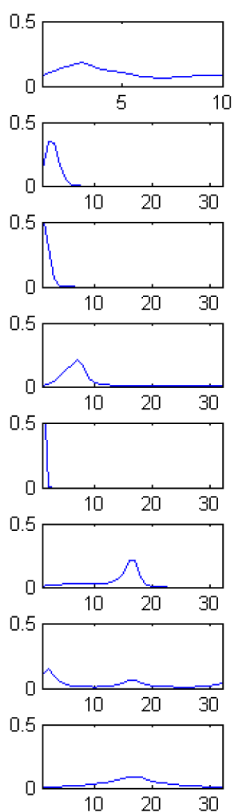

Figure 3: Feature histograms used to construct the 234-dimensional feature vectors are shown for each cell cycle phase for the PCNA labeled fluorescence channel. 
Table 3: Five-class recognition rates for individual features.

\begin{tabular}{|c|c|c|c|c|c|c|c|c|}
\hline & $\mathrm{R}$ & $\mathrm{I}$ & $\mathrm{GM}$ & $\mathrm{A} 1$ & $\mathrm{~A} 2$ & $\mathrm{~A} 3$ & $\mathrm{EV}$ & $\mathrm{HOG}$ \\
\hline \multirow{2}{*}{ CC (Channel 1) } & $52.35 \%$ & $65.10 \%$ & $56.04 \%$ & $59.40 \%$ & $45.30 \%$ & $58.39 \%$ & $67.45 \%$ & $46.98 \%$ \\
\cline { 2 - 8 } & \multicolumn{7}{|c|}{$82.55 \%$} \\
\hline \multirow{2}{*}{ PCNA (Channel 2) } & $48.32 \%$ & $32.89 \%$ & $34.23 \%$ & $67.79 \%$ & $29.87 \%$ & $66.44 \%$ & $72.82 \%$ & $50.00 \%$ \\
\cline { 2 - 6 } & \multicolumn{8}{|c|}{$91.61 \%$} \\
\hline \hline CC+PCNA & \multicolumn{8}{|c|}{$91 \%$} \\
\hline
\end{tabular}

Kyoto cell lines described in our previous work [9] where we were able to achieve similar cell cycle phase classification accuracies by using just 2D imagery (single slices), one fluorescence channel (PCNA) and fewer features (intensity and largest Hessian eigenvalue histograms). In the current work we did not have enough data to test the performance on identifying the mitosis phase. On the other hand, the discrimination of sub-phases within the S-phase is significantly improved compared to the previous results (confusion matrix in Table 1 of [9]).

\section{CONCLUSIONS}

We have shown that a large set of intensity, gradient, shape, and curvature-based features in combination with radial features can capture the subtle differences of foci patterns during the progression of cell cycle phases using dual channel 3D confocal microscopy. The classifier experiments of cell cycle phase recognition accuracy confirm that the overall classification performance is significantly better using dual channel imaging with a rich set of foci or blob distribution features extracted from the volumetric data. In particular, we are now able to discriminate more reliably between the subphases of S-phase; i.e. SE, SM, and SL, compared to the previous approach using only one PCNA channel $2 \mathrm{D}$ image. Having a more effective discrimination of cell cycle progression will enable quantitative studies of cellular processes in high-throughput studies involving large test series in future work. In addition to this, we plan to increase the recognition rates further by directly incorporating colocalization measures into the feature vector. Currently, the colocalization between chromocenters and replication structures is only captured implicitly by the dual channel radial features. A colocalization feature that is included explicitly in the feature vector should help the classifier to discriminate between the subphases of S-phase.

\section{ACKNOWLEDGMENTS}

Part of this work was funded by the US NIH NIBIB Award R33-EB00573 to K.P. and by grants from the Deutsche Forschungsgemeinschaft (DFG) to M.C.C.

\section{REFERENCES}

[1] T. Buck, A. Rao, L. Coelho, M. Fuhrman, J. Jarvik, P. Berget, and R. Murphy. Cell cycle dependence of protein subcellular location inferred from static, asynchronous images. In Int. Conf. of the IEEE Engineering in Medicine and Biology Society, volume 1, pages 1016-1019, 2009.

[2] F. Bunyak and K. Palaniappan. Efficient segmentation using feature-based graph partitioning active contours.
In 2009 IEEE 12th International Conference on Computer Vision, pages 873-880. IEEE, 2010.

[3] F. Bunyak, K. Palaniappan, V. Chagin, and M. Cardoso. Cell segmentation in time-lapse fluorescence microscopy with temporally varying sub-cellular fusion protein patterns. In Int. Conf. of the IEEE Engineering in Medicine and Biology Society, pages 1424-1428, 2009.

[4] F. Bunyak, K. Palaniappan, S. K. Nath, T. Baskin, and G. Dong. Quantitative cell motility for in vitro wound healing using level set-based active contour tracking. In Proc. $3^{\text {rd }}$ IEEE Int. Symp. Biomed. Imaging (ISBI), pages 1040-1043. April 2006.

[5] N. Dalal and B. Triggs. Histograms of oriented gradients for human detection. In IEEE Conf. Comp. Vision Patt. Recog., volume 1, pages 886-893, 2005.

[6] D. Demandolx and J. Davoust. Multicolour analysis and local image correlation in confocal microscopy. Journal of Microscopy, 185(1):21-36, 1997.

[7] O. Dzyubachyk, J. Essers, W. van Cappellen, C. Baldeyron, A. Inagaki, W. Niessen, and E. Meijering. Automated analysis of time-lapse fluorescence microscopy images: From live cell images to intracellular foci. Bioinformatics, 26(19):2424-2430, 2010.

[8] O. Dzyubachyk, W. Niessen, and E. Meijering. Advanced level-set based multiple-cell segmentation and tracking in time-lapse fluorescence microscopy images. In J.-C. Olivo-Marin, I. Bloch, and A. Laine, editors, IEEE International Symposium on Biomedical Imaging: From Nano to Macro, pages 185-188. IEEE, Piscataway, NJ, 2008.

[9] I. Ersoy, F. Bunyak, V. Chagin, M. Cardoso, and K. Palaniappan. Segmentation and classification of cell cycle phases in fluorescence imaging. In Medical Image Computing and Computer-Assisted Intervention (MICCAI), pages 617-624, 2009.

[10] A. Frangi, W. Niessen, K. Vincken, and M. Viergever. Multiscale vessel enhancement filtering. In Medical Image Computing and Computer-Assisted Intervention (MICCAI), pages 130-137, 1998.

[11] N. Harder, F. Mora-Bermudez, and W. G. et al. Automatic analysis of dividing cells in live cell movies to detect mitotic delays and correlate phenotypes in time. Genome Research, 19(11):2113-2124, 2009.

[12] S. Jaeger, C. Casas-Delucchi, M. Cardoso, and K. Palaniappan. Dual channel colocalization for cell cycle analysis using 3D confocal microscopy. In 20th Int. Conf. on Pattern Recognition, pages 2580-2583, Istanbul (Turkey), 2010.

[13] R. Kerekes, S. Gleason, N. Trivedi, and D. Solecki. 
Automated 3-D tracking of centrosomes in sequences of confocal image stacks. In Int. Conf. of the IEEE Engineering in Medicine and Biology Society, volume 1, pages 6994-6997, 2009.

[14] D. Knowles, D. Sudar, C. Bator-Kelly, M. Bissell, and S. Lelièvre. Automated local bright feature image analysis of nuclear protein distribution identifies changes in tissue phenotype. Proceedings of the National Academy of Sciences, 103(12):4445-4450, 2006.

[15] H. Leonhardt, H. Rahn, P. Weinzierl, A. Sporbert, T. Cremer, D. Zink, and M. Cardoso. Dynamics of DNA replication factories in living cells. Journal of Cell Biology, 149(2):271, 2000.

[16] R. Martin, S. Görisch, H. Leonhardt, and M. Cardoso. An unexpected link between energy metabolism, calcium, chromatin condensation and cell cycle. Cell Cycle, 6(19):2422-2424, 2007.

[17] E. Meijering, O. Dzyubachyk, I. Smal, and W. van Cappellen. Tracking in cell and developmental biology. In Seminars in Cell and Developmental Biology, pages 894-902. Elsevier, 2009.

[18] A. Mosig, S. Jaeger, C. Wang, S. Nath, I. Ersoy, K. Palaniappan, and S. Chen. Tracking cells in life cell imaging videos using topological alignments. Algorithms for Molecular Biology, 4(1):10, 2009.

[19] S. Nath and K. Palaniappan. Fast graph partitioning active contours for image segmentation using histograms. EURASIP Journal on Image and Video Processing, 9p., 2009.

[20] D. Padfield, J. Rittscher, N. Thomas, and B. Roysam. Spatio-temporal cell cycle phase analysis using level sets and fast marching methods. Medical Image Analysis, 13(1):143-155, 2009.

[21] K. Palaniappan, F. Bunyak, P. Kumar, I. Ersoy, S. Jaeger, K. Ganguli, A. Haridas, J. Fraser, R. Rao, and G. Seetharaman. Efficient feature extraction and likelihood fusion for vehicle tracking in low frame rate airborne video. In 13th Int. Conf. Information Fusion, Edinburgh, UK, July 2010.

[22] K. Palaniappan, I. Ersoy, and S. K. Nath. Moving object segmentation using the flux tensor for biological video microscopy. In PCM, pages 483-493, 2007.

[23] A. Smallcombe. Multicolor imaging: the important question of co-localization. Biotechniques, 30(6):1240-1247, 2001

[24] A. Sporbert, A. Gahl, R. Ankerhold, H. Leonhardt, and M. Cardoso. DNA polymerase clamp shows little turnover at established replication sites but sequential de novo assembly at adjacent origin clusters. Molecular Cell, 10(6):1355-1365, 2002.

[25] M. Wang, X. Zhou, F. Li, J. Huckins, R. King, and S. Wong. Novel cell segmentation and online SVM for cell cycle phase identification in automated microscopy. Bioinformatics, 24(1):94-101, 2008.

[26] C. Westin, S. Maier, H. Mamata, A. Nabavi, F. Jolesz, and R. Kikinis. Processing and visualization for diffusion tensor MRI. Medical Image Analysis, $6(2): 93-108,2002$ 